\title{
Theoretical and Experimental Research on a Novel Method of Cluster Magnetorheological Finishing Based on Array Circular Holes Polishing Disk
}

Bin Luo ( $\boldsymbol{\nabla} 71087 @$ nchu.edu.cn )

Nanchang Hangkong University https://orcid.org/0000-0001-6235-9930

Qiusheng Yan

Nanchang Hangkong University

Jingfu Chai

Nanchang Hangkong University

Wenqing Song

Nanchang Hangkong University

Jisheng Pan

Nanchang Hangkong University

\section{Research Article}

Keywords: Magnetorheological finishing, Array circular holes, Shear force mode, Material removal rate model

Posted Date: November 23rd, 2021

DOI: https://doi.org/10.21203/rs.3.rs-1097405/v1

License: (c) (i) This work is licensed under a Creative Commons Attribution 4.0 International License.

Read Full License 


\title{
Title Page
}

\section{Theoretical and experimental research on a novel method of cluster magnetorheological finishing based on array circular holes polishing disk}

\author{
Bin Luo ${ }^{1 *}$, Qiusheng Yan ${ }^{2 *}$, Jingfu Chai ${ }^{1}$, Wenqing Song ${ }^{1}$, Jisheng Pan ${ }^{2}$ \\ (1 School of Aeronautical Manufacturing Engineering, Nanchang Hangkong University, Nanchang 330000, China \\ 2 School of Electromechanical Engineering, Guangdong University of Technology, Guangzhou 510006, China) \\ * Corresponding Author; E-mail: 71087@ nchu.edu.cn
}

Title: Theoretical and experimental research on a novel method of cluster magnetorheological finishing based on array circular holes polishing disk

\section{Author names and affiliations:}

1. Given name: Bin, Family name: Luo

Affiliations address: School of Aeronautical Manufacturing Engineering, Nanchang Hangkong University, Nanchang 330000, China

e-mail address: 71087@nchu.edu.cn

2. Given name: Qiusheng, Family name: Yan

Affiliations address: School of Electromechanical Engineering, Guangdong

University of Technology, Guangzhou 510006, China

e-mail address: qsyan@gdut.edu.cn

3. Given name: Jingfu, Family name: Chai

Affiliations address: School of Aeronautical Manufacturing Engineering, Nanchang Hangkong University, Nanchang 330000, China 
e-mail address: chaijingfu@nchu.edu.cn

4. Given name: Wenqing, Family name: Song

Affiliations address: School of Aeronautical Manufacturing Engineering, Nanchang

Hangkong University, Nanchang 330000, China

e-mail address: wqsong@nchu.edu.cn

5. Given name: Jisheng, Family name: Pan

Affiliations address: School of Electromechanical Engineering, Guangdong

University of Technology, Guangzhou 510006, China

e-mail address: panjisheng@gdut.edu.cn

\section{Corresponding author:}

Bin Luo,e-mail address: 71087@nchu.edu.cn 


\section{Cover Letter}

Bin Luo

School of Aeronautical Manufacturing Engineering, Nanchang Hangkong University, Nanchang 330000, China

71087@nchu.edu.cn

\section{Dear Editors:}

Please excuse me for taking some of your time.

On behalf of my co-authors, I am submitting the enclosed manuscript "Theoretical and experimental research on a novel method of cluster magnetorheological finishing based on array circular holes polishing disk" for your kind consideration of its suitability for publication in The International Journal of Advanced Manufacturing Technology. We believe this article may be of particular interest to the readers of your journal.

Background: With the development and application of new generation semiconductor materials, the single crystal diamond, GaN, SiC, sapphire and other materials with wider band gap have higher hardness, which puts forward new requirements and challenges for flexible polishing technology. In this paper, a new method of cluster magnetorheological finishing based on array circular holes polishing disk is proposed. By machining array circular holes on the surface of polishing disk, which effectively increases the polishing force and material removal rate of workpiece surface. The efficient and ultra-smooth planarization processing of optoelectronic wafers is realized, but it is necessary to establish a model of polishing shear force and material removal rate to guide the optimization of polishing disk surface structure and polishing process.

Procedures: Firstly, based on the tribology principle, the contact forms between carbonyl iron powder chain, abrasive and workpiece are analyzed. On this basic, A shear force model of solid particles in MRF fluid was established, and the material removal rate prediction model was established by combining the shear force model with the velocity model. Then, the effects of polishing disks with different circular hole diameters on polishing shear force and material removal rate are analyzed. Finally, by conducting test on two-inch $(50.8 \mathrm{~mm})$ single-crystal sapphire 
substrates. The correctness of the model of polishing shear force and material removal rate is verified by rotary dynamometer and repeated single factor experiments.

Results: The errors between theoretical and experimental values of polishing shear force and material removal rate are $8.8 \%$ and $10.8 \%$, respectively. The new magnetorheological finishing method can realize the efficient and ultra-smooth planarization of optoelectronic wafers. The established model can theoretically guide the optimization of the surface structure and polishing process of polishing disks.

Significances: The established model of polishing shear force and material removal rate can be used for cluster magnetorheological finishing based on array circular holes polishing disk, which can provide theoretical and technical support for optimisation of technological parameters and structures of polishing devices.

Statement: we have reviewed the final version of the manuscript and approve it for publication. To the best of our knowledge and belief, this manuscript has not been published nor accepted elsewhere, and it is not being submitted to any other journal. We further confirm that all authors have checked the manuscript and have agreed to the submission.

\section{And the question to three answers is shown as below:}

\section{(1) What is your main contribution to the field?}

In order to further improve the polishing efficiency of hard and brittle materials, it is necessary to optimize the traditional cluster magnetorheological finishing method, so as to realize the ultrasmooth and flat processing of high hardness materials such as $\mathrm{SiC}, \mathrm{GaN}$, sapphire and so on. The main content of this paper is to put forward a new magnetorheological polishing method, and establish the corresponding polishing shear force and material removal rate model. So, based on the above researches, optimized parameters and the corresponding simulation model can be provided for the magnetorheological finishing.

\section{(2) What is novel? In theory, in experimental techniques, or a combination of both?}

The innovation of this research is that a new magnetorheological finishing method is proposed, and the polishing shear force and material removal rate model of this method are established, which provides guidance for the optimization of polishing process parameters and surface structure of polishing disk. 
(3) Does your paper have industrial applications? If yes, who are the likely user?

These achievements have been used in the ultra-smooth and planarization processing of optoelectronic wafers. In addition, Guangdong Nanotechnology Co., Ltd. is commercializing the above research.

If you have any queries, please don't hesitate to contact us.

Thank you very much for your time and consideration.

Yours Sincerely,

Bin Luo 


\title{
Theoretical and experimental research on a novel method of cluster magnetorheological finishing based on array circular holes polishing disk
}

\author{
Bin Luo $^{1 *}$ Qiusheng Yan ${ }^{2 *}$ Jingfu Chai ${ }^{1}$ Wenqing Song ${ }^{1}$ Jisheng Pan ${ }^{2}$
}

\begin{abstract}
: with the high performance of microelectronic and optoelectronic devices, the new generation of optoelectronic wafers is developing in the direction of large size and ultra-thinning, which requires ultra-smooth surfaces with sub-nanometer surface roughness. It puts forward new requirements and challenges for the efficient and ultra-smooth planarization processing of optoelectronic wafers. This paper proposes a novel method of cluster magnetorheological finishing based on array circular holes polishing disk, which can effectively improve the polishing shear force and polishing efficiency. The appropriate polishing shear force and material removal rate are the keys to achieve low roughness and low damage processing of optoelectronic wafers. Therefore, the shear force model of solid particles in magnetorheological finishing fluid is established based on the tribological principle. The material removal rate model is established by combining the polishing shear force model with the velocity model. The correctness of the above model is verified by the rotary dynamometer and repeated single-factor experiments. The errors between theoretical and experimental values of polishing shear force and material removal rate are $8.8 \%$ and $10.8 \%$, respectively. The new magnetorheological finishing method can realize the efficient and ultra-smooth planarization of optoelectronic wafers. The established model can theoretically guide the optimization of the surface structure and polishing process of polishing disks.
\end{abstract}

Keywords: Magnetorheological finishing, Array circular holes, Shear force mode, Material removal rate model

\section{Introduction*}

With the rapid development of microelectronics and optoelectronic technology, the high-performance requirements of microelectronic and optoelectronic devices, a new generation of optoelectronic wafers such as single crystal diamond, $\mathrm{GaN}, \mathrm{SiC}$, sapphire and so on are developing in the direction of large size and ultra-thin, which requires ultra-smooth surfaces with sub-nanometer surface roughness [1]. Therefore, new requirements and challenges are put forward for flexible polishing technology. As a deterministic flexible polishing method, magnetorheological finishing (MRF) technology has many remarkable advantages, such as wide

Bin luo

71087@nchu.edu.cn

1 School of Aeronautical Manufacturing Engineering, Nanchang Hangkong University, Nanchang 330000, China application range, high machining precision, high convergence efficiency, small subsurface damage and so on [2]. The processing principle is that the magnetic particles in the MRF fluid are serialized to form a viscoelastic Bingham flexible polishing pad, and the magnetic particle chain wraps and clamps the abrasive particles to remove the surface material of the workpiece [3].

For the flattening process of hard and brittle materials, the disk-MRF method proposed by some scholars can effectively solve the processing efficiency problem. The magnetic field generator of disk-MRF is the critical component of MRF, which has an important influence on polishing efficiency and polishing quality. By establishing the mathematical model of the flux density distribution of cluster permanent magnets, Meng et al. [4] studied the effects of different distribution modes of 
magnets on polishing efficiency and uniformity. Luo et al. [5] proposed a high-efficiency and high-quality MRF method using the permanent magnet excitation unit with a straight air gap, which realized supersmooth surface planarization of zirconia ceramics with surface roughness less than Ra $1 \mathrm{~nm}$. Wu et al. [6] proposed a new method of disk-MRF with a low frequency alternating magnetic field. The effects of current frequency, working gap, slot speed and workpiece speed on surface roughness and material removal rate were studied. The surface roughness of K9 glass decreased from Ra $567.08 \mathrm{~nm}$ to $\mathrm{Ra} 9.63 \mathrm{~nm}$ after being polished for $120 \mathrm{~min}$. Guo et al. [7] studied the effects of three process parameters, such as magnet speed, MCF carrier speed and working gap, on pressure, shear stress and material removal rate. A material removal rate model combining positive pressure and shear stress is proposed. This model mainly determines the material removal rate by shear stress, not by positive pressure. In order to further improve the polishing efficiency and uniformity of hard and brittle materials, Xiu et al. [8] proposed a new method of reciprocating MRF and analyzed the micro-removal mechanism and characteristics of reciprocating swing polishing. After polishing 40min of borosilicate glass (K9), the surface roughness decreased from $\mathrm{Ra} 408 \mathrm{~nm}$ to $\mathrm{Ra} 15 \mathrm{~nm}$. Xu et al. [9] proposed the cluster magnetorheological-porous foam finishing method (CMRFF). Compared with CMRF, CMRFF can obtain a higher normal force. After polishing $45 \mathrm{~min}$ with the silicon wafer, the surface roughness of CMRFF decreased from $\mathrm{Ra}$ $49.66 \mathrm{~nm}$ to $\mathrm{Ra} 16.991 \mathrm{~nm}$, while the surface roughness of CMRF decreased from Ra $50.559 \mathrm{~nm}$ to $\mathrm{Ra} 22.891 \mathrm{~nm}$. Zhai et al. [10] put forward the method of ultrasonic-assisted MRF and established the mathematical model of shear force $F_{\tau}$ and normal force $F_{\mathrm{n}}$ on the surface of sapphire. Both $F_{\tau}$ and $F_{\mathrm{n}}$ increase with the increase of abrasive volume concentration and decrease with the increase in excitation and working gaps. Ghai et al. [11] put forward the method of chemical mechanical MRF. Through the chemical reaction-assisted machining on the aluminium alloy surface, the ultra-smooth surface of Ra $0.597 \mathrm{~nm}$ can be obtained.

The research group proposes a cluster MRF method for "region" processing, which forms an MRF pad in real-time, effectively controls the micro removal process of planarization processing and realizes large-area plane high precision deterministic ultra-smooth machining [12-14]. K9 glass, single crystal silicon and $6 \mathrm{H}-\mathrm{SiC}$ were carried out by cluster MRF for $30 \mathrm{~min}$. The surface roughness of K9 glass decreased from $\mathrm{Ra} 340 \mathrm{~nm}$ to $\mathrm{Ra} 1.4 \mathrm{~nm}$, the single-crystal silicon wafer decreased from Ra $57.2 \mathrm{~nm}$ to $\mathrm{Ra} 4 \mathrm{~nm}$, and the single-crystal $\mathrm{SiC}$ decreased from $\mathrm{Ra} 72.89 \mathrm{~nm}$ to $\mathrm{Ra} 1.9 \mathrm{n} \mathrm{m}$. The high processing efficiency and nanometer roughness surface can be obtained, which shows that cluster MRF is feasible as an efficient ultra-precision planarization method. However, for the processing of photoelectric materials with high hardness, such as sapphire and $\mathrm{SiC}, \mathrm{GaN}$. The low material removal rate is a problem to be solved. Luo et al. [15] proposed a processing method to control the Bingham fluid characteristics of the MRF pad. Compared with MRF, the material removal rate increases by $107 \%$, and the surface roughness is reduced by $11 \%$. The surface roughness value can be reduced from $\mathrm{Ra} 5.14 \mathrm{~nm}$ to $\mathrm{Ra} 0.24 \mathrm{~nm}$ by optimizing the process parameters. The above research mainly optimizes the polishing device from the aspects of magnetic field generator and energy field-assisted processing. There is little research on the influence of disk structure on the effect of MRF, and the polishing shear force model and material removal rate model of the new cluster MRF method based on array circular holes polishing disk has not been established and studied. 
This paper establishes the polishing shear force model of a novel cluster MRF method based on array circular holes polishing disk according to the tribological principle. The influence of hole diameter on polishing shear force was measured by the rotary dynamometer, the correctness of the mechanical model was verified, and the material removal rate model was established by the velocity and polishing shear models. The single-crystal sapphire polishing experiment confirmed the feasibility of material removal model prediction.

\section{Principle and polishing shear force model of cluster MRF based on array circular holes polishing disk}

\subsection{Principle of cluster MRF based on array circular holes polishing disk}

Figure 1 shows the principle of cluster MRF based on array circular holes polishing disk. Under the action of the annular uniformly distributed magnetic field, the carbonyl iron powder in the MRF fluid forms a closely arranged magnetic chain string, and the rotating motion of the polishing disk and the workpiece drive the abrasive particles wrapped and held by the magnetic chain to plough and remove the workpiece surface. At the same time, the abrasive particles in contact with the workpiece polishing disk surface will also remove the polishing disk surface. The circular holes arranged by the array is processed on the surface of the polishing disk, which makes the carbonyl iron powder chain string embedded in the circular holes to form a "pinning" effect, which reduces the relative slip between the polishing pad and the polishing disk and enhances the fixation force between the polishing pad and the polishing disk. The number of magnetic particles and abrasives in the processing area is significantly increased, and the force of the polishing pad acting on the surface of the workpiece is enhanced.

Figure 2 (a) shows an experimental device of cluster MRF based on array circular holes polishing disk, which has five servo motions of $\mathrm{X}, \mathrm{Y}, \mathrm{Z}, \mathrm{C} 1$ and C2. Six cylindrical permanent magnets are placed under the array circular holes polishing disk or smooth polishing disk (Fig. 2 (c)). The magnetic field distribution is shown in Fig. (f), showing a bimodal distribution. Six cylindrical magnets are evenly distributed around the circle with a diameter of $10 \mathrm{~mm}$, as shown in Fig. 2 (d). The carbonyl iron powder in the MRF fluid can be formed by a single magnetic pole to form a flexible micro-grinding head with a diameter of about $50 \mathrm{~mm}$. Six flexible microgrinding heads form a single ring polishing pad, and the lower end of the polishing pad is magnetically adsorbed on the surface of the array circular holes polishing disk. As shown in Fig. 2 (b), the abrasive at the upper end of the polishing pad is flexibly clamped by a carbonyl iron powder chain to process the workpiece surface, as shown in Fig. 2 (e).

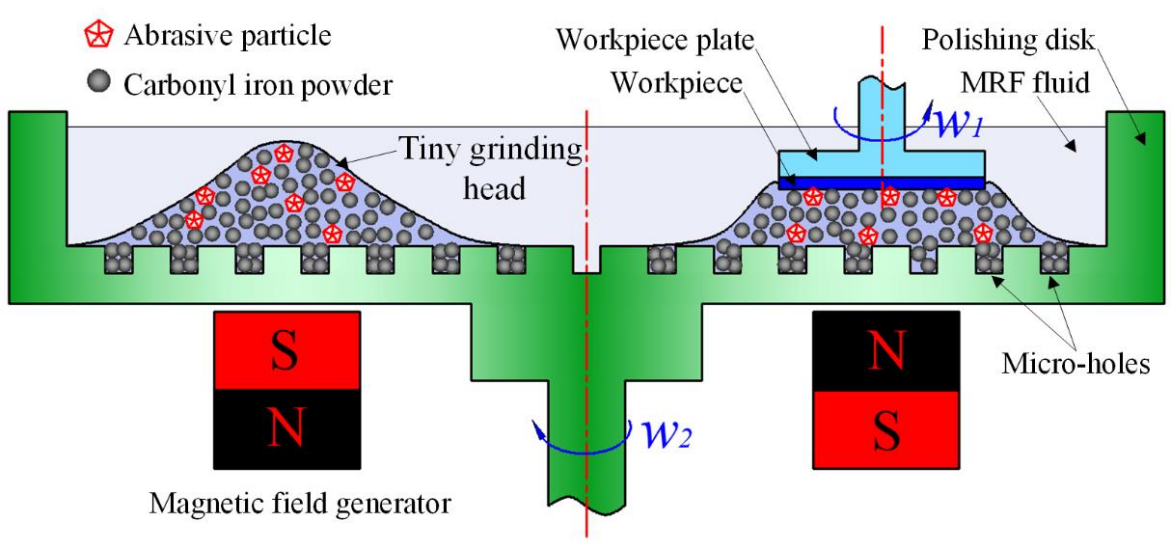

Fig. 1 principle of cluster MRF based on array circular holes polishing disk 

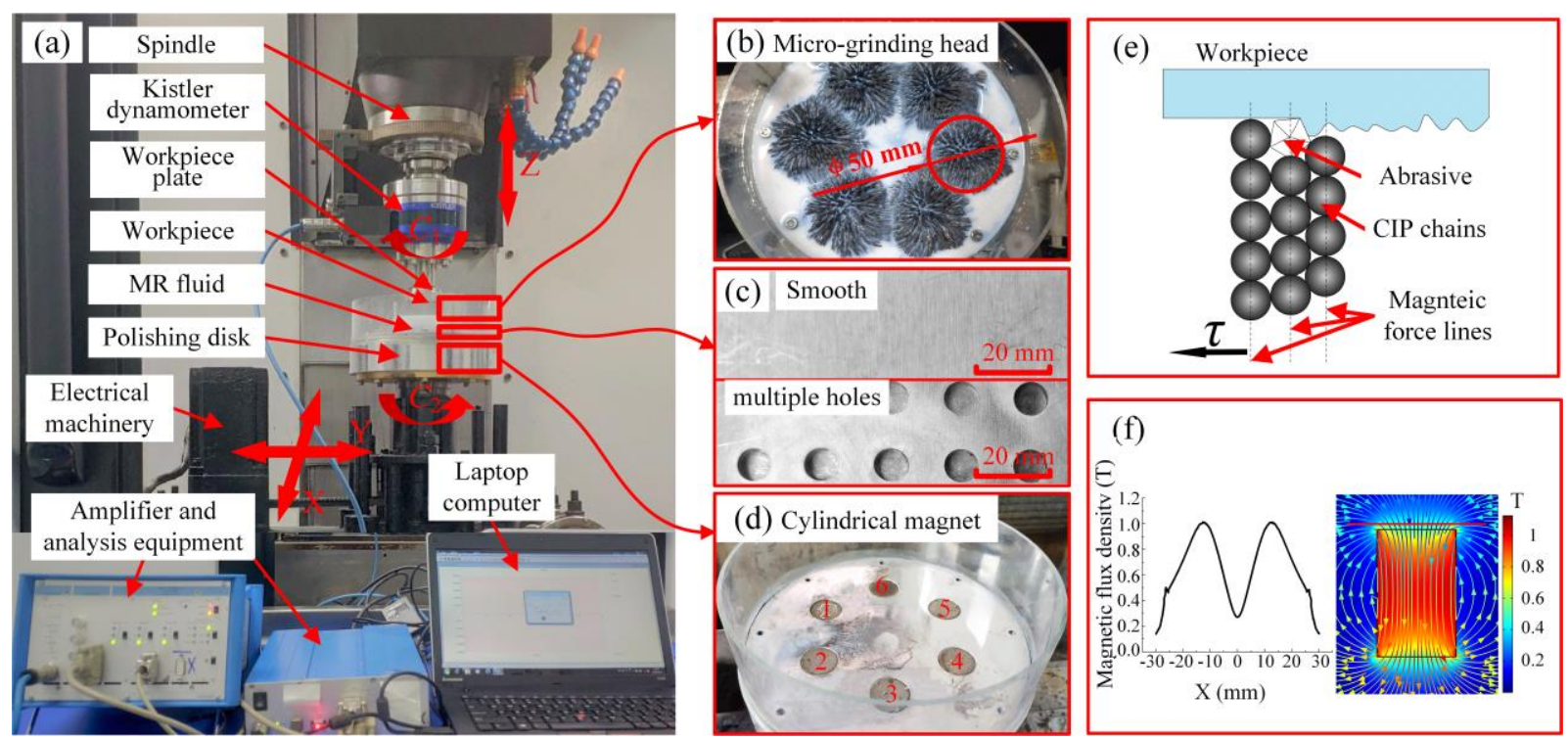

Fig. 2 Experimental device for cluster MRF based on array circular holes polishing disk

\subsection{Establishment of polishing shear force model of cluster MRF based on array circular holes polishing disk}

In the process of cluster MRF based on array circular holes polishing disk, the fixing force between the polishing pad and the polishing disk is strengthened because of the consolidation effect of circular holes on the surface of polishing disk. The relative slip between the polishing pad and the polishing disk surface is reduced, and the polishing shear force of the polishing pad acting on the workpiece surface is enhanced. The polishing shear force on the surface of the workpiece can be divided into chain yield shear force and friction shear force because the carbonyl iron powder chain embedded in the array circular holes is fixed, which provides the chain yield shear force in the machining process. The friction shear force caused by abrasive, carbonyl iron powder and rough workpiece surface is mainly divided into friction shear force caused by abrasive and carbonyl iron powder ploughing effect, friction shear force caused by adhesion between MRF fluid and workpiece surface, friction shear force produced by the rough surface.
The sum of yield shear stress and friction shear stress of carbonyl iron powder chain is the total shear stress:

$$
\tau_{\text {total }}=\tau_{m}+\tau_{\text {tribo }}
$$

\subsubsection{Yield shear stress $\tau_{m}$ of carbonyl iron} powder chain

Lopez et al. [16] have studied that the yield shear stress of magnetic particles under the action of the magnetic field is related to its deflection angle $\theta$. The relationship is $\gamma=\tan \theta$. The yield shear stress can be expressed as follows:

$$
\tau_{z x}=E \delta_{z x}+\frac{\partial E^{0}}{\partial \gamma_{z x}}+\frac{1}{2}\left(H_{z} B_{x}+H_{x} B_{z}\right)
$$

Where, $\boldsymbol{\gamma}_{z x}$ and $\boldsymbol{\delta}_{z x}$ represents the strain tensor and the unit tensor, respectively. The energy function $E^{\beta}$ is as follows [17]:

$$
E^{O} \equiv E-\mathbf{H} \cdot \mathbf{B}=E_{0}-\int_{0}^{H} \mathbf{B} d \mathbf{H}^{G 0}
$$

Where, $E_{0}$ represents the free energy without the magnetic field. Lopez et al. [16] have shown that only the third term in Equation (2) significantly affects the yield shear stress, while the other two terms can be ignored. The permeability is divided 
into the permeability parallel $\mu_{\mathrm{P}}$ and the permeability perpendicular $\mu_{\perp}$ to the direction of the chain. Assuming that the permeability does not depend on the strength of the magnetic field, it can be obtained by calculation:

$$
\begin{aligned}
& E \cong E_{0}-\int_{0}^{H} \mu_{0} \mu \mathbf{H} d \mathbf{H} \\
& =E_{0}-\int_{0}^{H_{\mathrm{P}}} \mu_{0} \mu_{\mathrm{P}} H_{\mathrm{P}} d H-\int_{0}^{H_{\perp}} \mu_{0} \mu_{\perp} H_{\perp} d H \\
& =E_{0}-\frac{1}{2} \mu_{0} \mu_{\mathrm{P}} H_{\mathrm{P}}^{2}-\mu_{0} \mu_{\perp} H_{\perp}^{2}
\end{aligned}
$$

The $\mu_{\mathrm{P}}=H \cos \theta \quad, \quad \mu_{\perp}=H \sin \theta \quad$ and trigonometric function equations $\cos ^{2} \theta=\frac{1}{1+\gamma^{2}}$ and $\sin ^{2} \theta=\frac{\gamma^{2}}{1+\gamma^{2}}$ are converted into Equation 4 to obtain:

$E^{\circ}=E_{0}-\frac{1}{2} \mu_{0} \mu_{\mathrm{P}} H^{2} \frac{1}{1+\gamma^{2}}-\frac{1}{2} \mu_{0} \mu_{\perp} H^{2} \frac{\gamma^{2}}{1+\gamma^{2}}(5)$

By substituting Equation (5) into Equation (2), the yield shear stress of carbonyl iron powder chain is obtained as follows:

$$
\tau_{m}=-\frac{1}{2} \mu_{0} H^{2}\left[\frac{\partial \mu_{\mathrm{P}}}{\partial \gamma} \frac{1}{1+\gamma^{2}}+\frac{\partial \mu_{\perp}}{\partial \gamma} \frac{\gamma^{2}}{1+\gamma^{2}}\right]
$$

The relationship between yield shear stress $\tau_{m}$ and shear strain $\gamma$ of carbonyl iron powder chain is described by Equation (6). In order to obtain the relationship between the shear strain $\gamma$ and the workpiece rotation speed, Hegger et al. [17] established the relationship between the rotation speed of the rotator and the shear strain as follows:

$$
\gamma(\omega)=16.10^{-5}+8.141 \cdot 10^{-5} \cdot \omega+8.105 \cdot 10^{-9} \cdot \omega^{2}(7)
$$

In order to calculate the shear yield stress, it is necessary to obtain the magnetic permeability $\mu_{\mathrm{P}}$ and $\mu_{\perp}$. Lopez et al. [16] proposed the relationship between shear strain $\gamma$ and particle distance $L$ as follows:

$$
\frac{L}{R_{\text {Iron }}}=2\left(\sqrt{1+\gamma^{2}}-1\right)
$$

$R_{\text {Iron }}$ represents the particle size of carbonyl iron powder, which is $3 \mu \mathrm{m}$. Hegger et al. [17] studied the effects of different particle gaps on parallel permeability $\mu_{\mathrm{P}}$ and perpendicular permeability $\mu_{\perp}$ and established the functional relationship between the two kinds of permeability as follows:

$$
\left\{\begin{array}{l}
\mu_{\mathrm{P}}(L)=3.22 \cdot e^{-2.26 L}+4.45 \cdot e^{-0.049 L} \\
\mu_{\perp} \approx 3.1
\end{array}\right.
$$

Because $\mu_{\perp}$ is an approximate value, Equation (6) can be simplified to:

$$
\tau_{m}=-\frac{1}{2} \mu_{0} H^{2} \frac{\partial \mu_{\mathrm{P}}}{\partial \gamma} \frac{1}{1+\gamma^{2}}
$$

After substituting Equation (8) into Equation (9), $\frac{\partial \mu_{\mathrm{P}}}{\partial \gamma}$ is obtained as follows:

$$
\begin{aligned}
& \frac{\partial \mu_{\mathrm{P}}}{\partial \gamma}=\frac{2 R_{\text {Iron }} \gamma}{\sqrt{\gamma^{2}+1}}\left(-7.28 \cdot e^{-4.52 R_{\text {Iron }}\left(\sqrt{\gamma^{2}+1}-1\right)}\right. \\
& \left.-0.218 \cdot e^{-0.098 R_{\text {Iron }}\left(\sqrt{\gamma^{2}+1}-1\right)}\right)
\end{aligned}
$$

\subsubsection{Friction shear stress $\tau_{\text {tribo }}$}

The mathematical model of the total friction force is established, as follows:

$$
F_{R}=F_{p}+F_{a}+F_{r}
$$

Where, $F_{\mathrm{p}}$ represents the friction caused by the ploughing effect of abrasive and carbonyl iron powder, $F_{\text {a }}$ represents the friction caused by the adhesion of MRF fluid to the workpiece surface, and $F_{\mathrm{r}}$ represents the friction caused by the rough surface. 


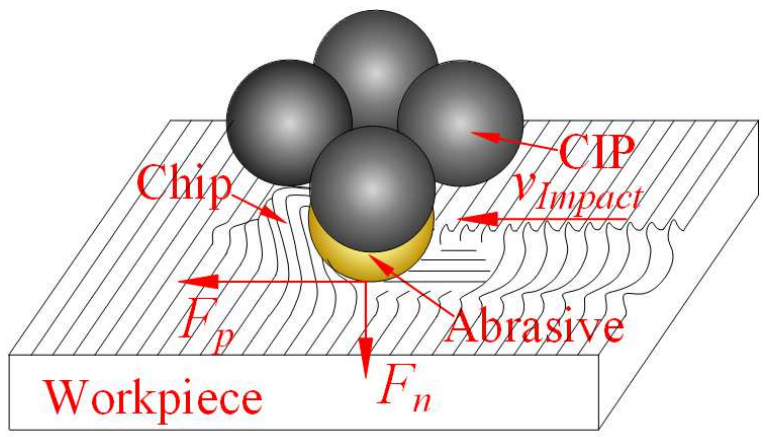

Fig. 3 Force analysis diagram in the process of abrasive particle processing

When the carbonyl iron powder chain flexibly holds the abrasive particles to process the workpiece surface, the shear force $F_{\mathrm{p}}$ and positive pressure $F_{\mathrm{n}}$ produced by the abrasive particles or carbonyl iron powder on the workpiece surface are shown in Fig. 3. $F_{\mathrm{p}}$ is the friction force caused by ploughing effect, which can be divided into friction force $F_{\mathrm{p} 1}$ generated by abrasive pressing into the workpiece surface for plastic removal. The other part is $F_{\mathrm{p} 2}$ caused by elastic pressing between carbonyl iron powder particles and the workpiece surface. A single abrasive and carbonyl iron powder are pressed into the workpiece surface under positive force $F_{\mathrm{pn}}$. Using the Hertz contact model, the diameter $w_{\text {Iron }}$ and $w_{\text {Abra }}$ of the abrasive and carbonyl iron powder pushed into the actual contact area of the workpiece surface can be calculated [18]:

$$
w=2 \cdot \sqrt{\frac{R^{*}}{\pi E^{*}}} \cdot \sqrt{F_{p n}}
$$

Where $E^{*}$ represents Young's modulus and $R^{*}$ represents relative radius, the calculation formula is as follows:

$$
\begin{gathered}
E^{*}=\left(\frac{1-v^{2}}{E}+\frac{1-v_{w}^{2}}{E_{w}}\right)^{-1} \\
R^{*}=\left(\frac{1}{R^{\prime}}+\frac{1}{R_{r o}}\right)^{-1}
\end{gathered}
$$

Where $v$ represents the Poisson's ratio of the abrasive particles or carbonyl iron powder, $E$ represents Young's modulus of the abrasive particles or carbonyl iron powder, $v_{\mathrm{w}}$ and $E_{\mathrm{w}}$ represent the Poisson's ratio and Young's modulus of the workpiece, $R^{\prime}$ represents the particle size of the abrasive particles or carbonyl iron powder, and $R_{r o}$ represents the radius of the assumed roughness peak. The depth $d_{\text {Iron }}$ and $d_{\text {Abra }}$ of abrasive particles and carbonyl iron powder pressed into the surface of the workpiece can be calculated as follows:

$$
d=\frac{w^{2}}{2 R^{*}}
$$

Carbonyl iron powder produces a ploughing effect on the workpiece surface. It plastically removes the material, $\sigma_{\max }$ represents $100 \%$ of the yield strength of the workpiece material, and the friction force $F_{\mathrm{p} 1}$ is caused by the contact between the abrasive and the workpiece can be calculated as [19]:

$$
F_{p 1}=1.6 \pi R_{\text {Iron }} \cdot d_{\text {Iron }} \cdot \sigma_{\max }
$$

Assuming that $\sigma_{\max }$ represents $80 \%$ of the yield strength of the workpiece material (tribological principle), the material undergoes elastic deformation under a load lower than the yield strength. Similarly, the friction force $F_{\mathrm{p} 2}$ of the contact surface between carbonyl iron powder and workpiece is:

$$
F_{p 2}=2 \pi R_{a b r a} \cdot d_{a b r a} \cdot \sigma_{\max }
$$

The friction force caused by the ploughing effect can be expressed as follows:

$$
F_{p}=\sigma_{\max } \cdot\left(1.6 \pi R_{\text {Iron }} \cdot d_{\text {Iron }}+2 \pi R_{a b r a} \cdot d_{a b r a}\right)
$$

$F_{\mathrm{a}}$ is the sum of the friction force $F_{\mathrm{a} 1}$ and $F_{\mathrm{a} 2}$ caused by the adhesion of abrasive particles and carbonyl iron powder to the rough workpiece surface. Shizhu et al. [20] proposed that the adhesion force model is:

$$
F_{a}=\pi \cdot\left(\frac{w}{2}\right)^{2} \cdot \tau_{\max }
$$


Where, $\tau_{\max }$ represents the shear yield stress of abrasive particles and carbonyl iron powder. The abrasive particles are not affected by the magnetic field, so that the shear yield stress $F_{\mathrm{a} 1}$ can be ignored.

$F_{\mathrm{r}}$ represents the friction force between abrasive particles, carbonyl iron powder and roughness surface $F_{\mathrm{r} 1}$ and $F_{\mathrm{r} 2}$, Berman et al. [21] put forward the corresponding model as follows:

$$
F_{r}=4 \pi \cdot w \cdot \frac{k \cdot \varepsilon_{i} \cdot \Delta D}{\Delta d \cdot D_{0}}
$$

Where, $k$ represents the surface energy of the material, $\Delta D$ represents the peak-valley value of the rough surface, $\Delta d$ represents the distance between the peak-valley values, and $D_{0}$ represents the average distance between the two peaks, as shown in Fig. 4. $\varepsilon_{i}$ represents a constant between $0<\varepsilon_{i}<1$, depending on the energy loss and transfer during friction.

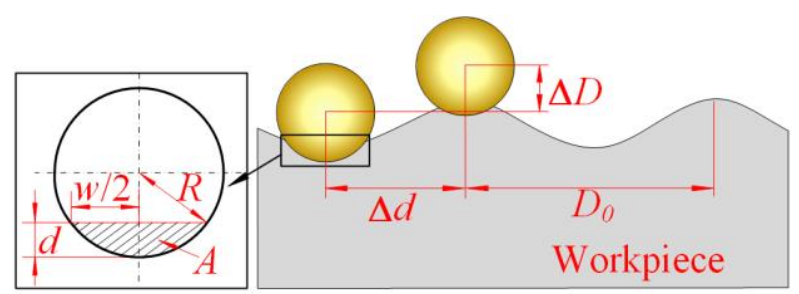

Fig. 4 interaction between abrasive particles and workpiece surface

The shear stress caused by friction force in MRF can be expressed as:

$$
\begin{aligned}
& \tau_{\text {tribo }}=\left(\frac{F_{p 1}+F_{r 1}}{A_{A b r a}}\right) \cdot N_{A b r a} \\
& +\left(\frac{F_{p 2}+F_{a 2}+F_{r 2}}{A_{\text {Iron }}}\right) \cdot N_{\text {Iron }}
\end{aligned}
$$

Where, $A_{\text {Abra }}$ and $A_{\text {Iron }}$ represent the contact area between abrasive and carbonyl iron powder and workpiece surface, respectively. $N_{\text {Abra }}$ and $N_{\text {Iron }}$ represent the number of particles contacted by abrasive and carbonyl iron powder with the workpiece, respectively. The calculation formula is as follows:

$$
\begin{gathered}
N_{\text {Abra }}=\frac{A_{\text {Abra }}}{S_{\text {Abra }}} \cdot \frac{\pi}{3 \sqrt{2}} \\
N_{\text {Iron }}=\frac{A_{\text {Iron }}}{S_{\text {Iron }}} \cdot \frac{\pi}{3 \sqrt{2}}
\end{gathered}
$$

Where, $S_{\text {Abra }}$ and $S_{\text {Iron }}$ represent the maximum cross-sectional area of abrasive particles and carbonyl iron powder, respectively.

\subsection{Establishment of material removal rate model of cluster MRF based on array circular holes polishing disk}

A large number of scholars have established the material removal rate function in the process of MRF. Lambropoulos et al. [22] put forward a material removal rate model based on the combination of polishing shear force model and velocity model by using shear force instead of positive force.

$$
M R R=C_{p} \cdot \frac{E_{w}}{K_{c} H_{v}^{2}} \cdot \tau_{\text {total }} \cdot V
$$

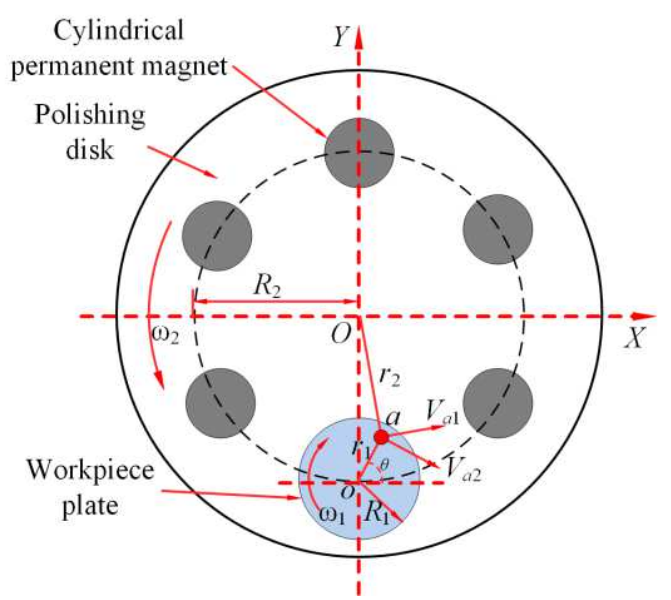

Fig.5 Motion analysis model of abrasive particles

Where, $K_{\mathrm{c}}$ is the fracture toughness of the photoelectric wafer, $H_{\mathrm{v}}$ is the Vickers hardness of the photoelectric wafer, $\tau_{\text {total }}$ represents the polishing shear force, $V$ represents the movement speed of the abrasive particles, and the motion model of the abrasive particles is shown in Fig. 5 . 
In the polishing process, the rotational speed of the polishing disk is $\omega 2$. Based on the polishing disk coordinate system XOY, the velocity motion formula of any point a on the polishing pad relative to the coordinate origin $\mathrm{O}$ point is established as follows:

$$
V_{a 1}=\omega_{2} \cdot r_{2}
$$

The rotational speed of the workpiece disk is $\omega_{1}$. Based on the workpiece coordinate system xoy, the velocity motion formula of any point a on the workpiece surface relative to the coordinate origin o point is established as follows:

$$
V_{a 2}=\omega_{1} \cdot r_{1}
$$

The velocity of any point on the workpiece in the coordinate system XOY can be expressed as:

$$
V_{a}=V_{a 1}+V_{a 2}=\omega_{1} r_{1}+\omega_{2} r_{2}
$$

According to the geometric relationship shown in Fig. 5, it can be concluded that:

$$
r_{2}^{2}=r_{1}^{2}+R_{2}^{2}-2 r_{1} R_{2} \cos (\pi-\theta)
$$

The conversion formula between velocity and angular velocity is as follows:

$$
\omega=2 \pi n
$$

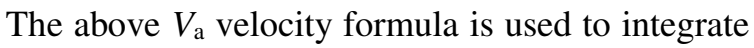
the region of the workpiece surface, and the average velocity of the workpiece surface is expressed as follows:

$$
\begin{aligned}
& V=\iint_{D} V_{a} d A=\frac{2 \pi n_{2}}{60}\left(\pi R_{1}^{2}+2 R_{1} R_{2}\right) \\
& +\frac{2 \pi n_{1}}{60} \pi R_{1}^{2}
\end{aligned}
$$

\section{Experimental equipment and conditions}

\subsection{Experimental equipment}

The array circular holes polishing disks with circular hole diameters of $\varnothing 1 \mathrm{~mm}, \varnothing 2 \mathrm{~mm}$, $\varnothing 4 \mathrm{~mm}$ and $\varnothing 5 \mathrm{~mm}$ were selected for experimental study. The array circular holes polishing disks of different diameters are installed on the surface of the polishing device shown in Fig. 2. The MRF force experimental testing device and dynamometer amplifier and analysis processor are shown in Fig. 2 . The Kistler 9171A rotary dynamometer is used to measure the MRF force. The rotary dynamometer is installed on the spindle through the adapter. The workpiece is fixed on the testing station at the bottom of the dynamometer. When testing the polishing force, the workpiece surface is in contact with the polishing pad by adjusting the machining gap to the cutter block. The polishing force measurement in the polishing process is realized under the rotating motion of the workpiece and the polishing disk.

\subsection{Experimental equipment}

The experimental workpiece is a 2-inch singlecrystal sapphire substrate with an original thickness of $350 \mu \mathrm{m}$ and a surface roughness of Ra $3.23 \mathrm{~nm}$, as shown in Fig. 6. The experiments are carried out with different circular hole diameter polishing disks, and each experiment is repeated three times. The processing parameters are shown in Table 1 . The slurry used in the experiment is water-based MRF fluid, and the abrasive is silica sol. The surface roughness of single-crystal sapphire was measured, and its surface morphology was observed by ContourGT-X white light interferometer. The average value of the measurement was regarded as the average roughness of single-crystal sapphire. The quality of sapphire substrate before and after processing is measured by GB204 precision electronic balance (measuring accuracy is $0.1 \mathrm{mg}$ ), and the material removal rate is calculated by formula. 


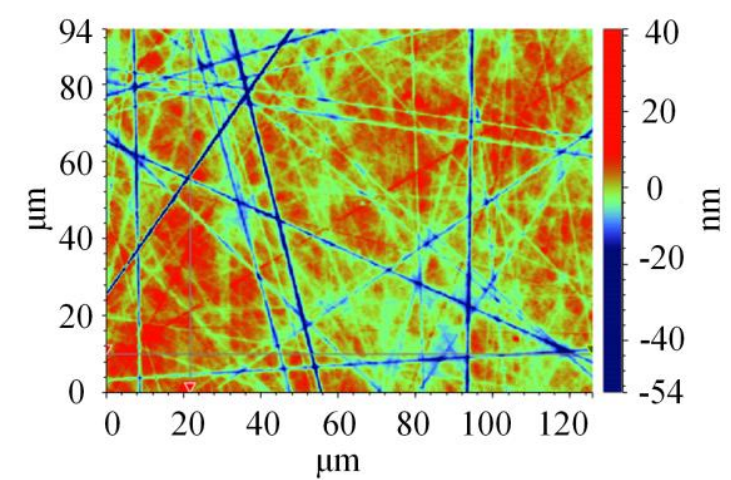

Fig. 6 Original surface morphology of workpiece

Table 1 level of processing parameters

\begin{tabular}{cc}
\hline processing parameters & level \\
\hline Circular hole diameter $(\mathrm{mm})$ & $1,2,3,4,5$ \\
Machining gap $(\mathrm{mm})$ & 1 \\
Polishing disk rotational speed $(\mathrm{r} / \mathrm{min})$ & 40 \\
Workpiece rotational speed $(\mathrm{r} / \mathrm{min})$ & 350 \\
\hline
\end{tabular}

\section{Experimental results and analysis}

\subsection{Influence of circular hole diameter on polishing Shear Force}

In order to verify the correctness of the shear force model of MRF based on array circular holes polishing disk, a 2-inch single-crystal sapphire was used to measure the shear force. Under the condition that the circular hole spacing is $10 \mathrm{~mm}$ and the circular hole depth is $2 \mathrm{~mm}$. The polishing shear force is measured with different circular hole diameters: Ø $0 \mathrm{~mm}, \varnothing 1 \mathrm{~mm}, \varnothing 2 \mathrm{~mm}, \varnothing 3 \mathrm{~mm}, \varnothing 4$ $\mathrm{mm}, \varnothing 5 \mathrm{~mm}$. The measured results are shown in Fig. 7.

Figure 7 shows the influence of different circular hole diameters on the polishing shear force. In the process of MRF, a large polishing shear force appears at the initial stage of contact between the workpiece and the polishing pad, but with the gradual increase of processing time. The polishing shear force decreases rapidly and then tends to be stable. In the process of traditional cluster MRF (circular hole diameter is $0 \mathrm{~mm}$ ), the detected force signal is relatively stable, there is some noise interference, and the average shear force is about $2.66 \mathrm{~N}$, as shown in Fig. 7 (a). Under the condition of cluster MRF based on array circular holes polishing disk, the noise and amplitude of the detection force signal increase gradually. When the circular hole diameters are $1 \mathrm{~mm}, 1 \mathrm{~mm}, 2 \mathrm{~mm}, 3$ $\mathrm{mm}, 4 \mathrm{~mm}, 5 \mathrm{~mm}$, the corresponding average shear forces are $3.95 \mathrm{~N}, 4.27 \mathrm{~N}, 5.47 \mathrm{~N}, 6.02 \mathrm{~N}$ and 6.38 $\mathrm{N}$, respectively, as shown in Fig. 7. With the circular hole diameter increase, the polishing shear force on the workpiece surface increases gradually. When the circular hole diameter is $5 \mathrm{~mm}$, the polishing shear force increases the largest by $139.8 \%$ compared with the traditional cluster MRF. The main reason is that the larger the circular hole diameter on the polishing disk surface, the more carbonyl iron powder chains are embedded in the circular hole. It can effectively slow down the relative slip between the polishing disk and the polishing pad, so that the polishing shear force of the polishing pad acting on the workpiece surface is greatly improved. 


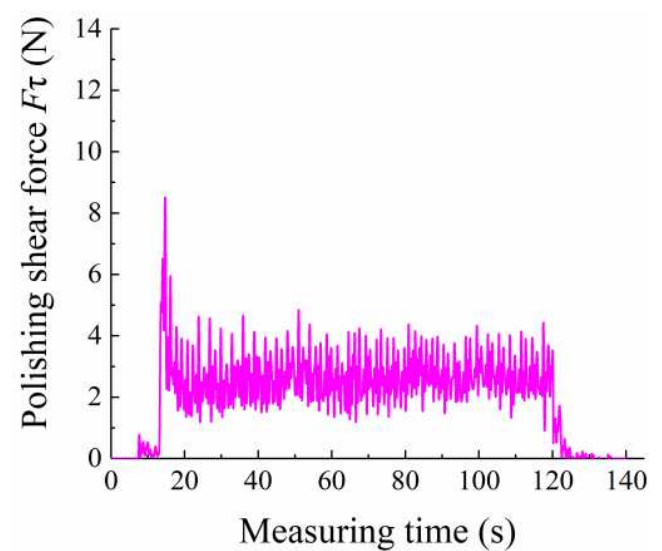

(a) $\varnothing 0 \mathrm{~mm}$

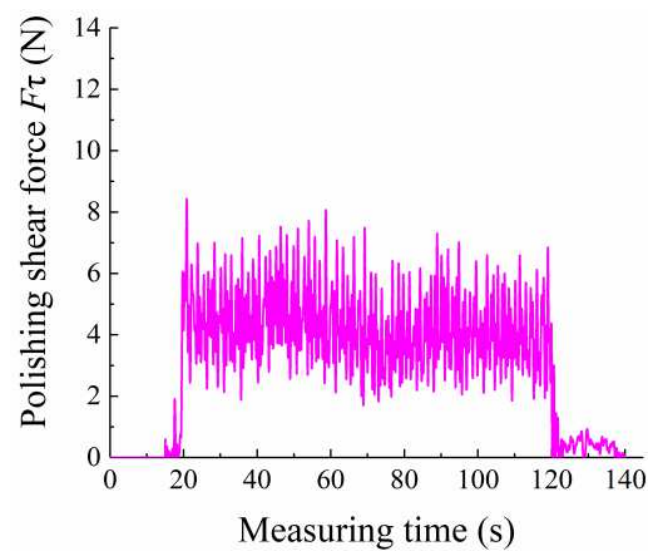

(c) $\varnothing 2 \mathrm{~mm}$

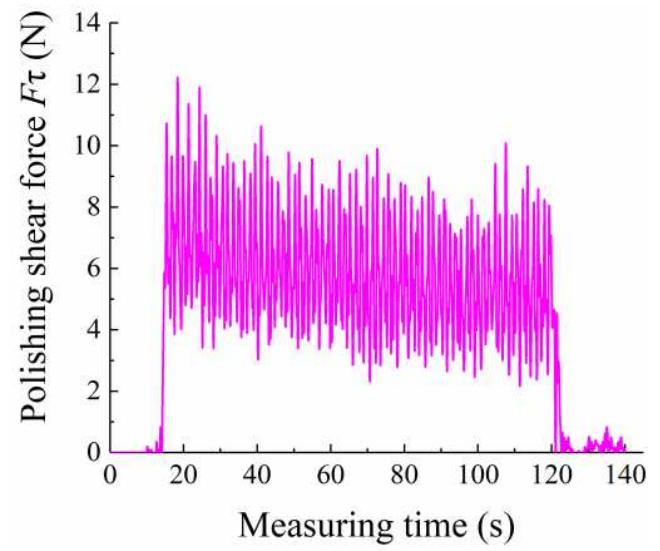

(e) $\varnothing 4 \mathrm{~mm}$

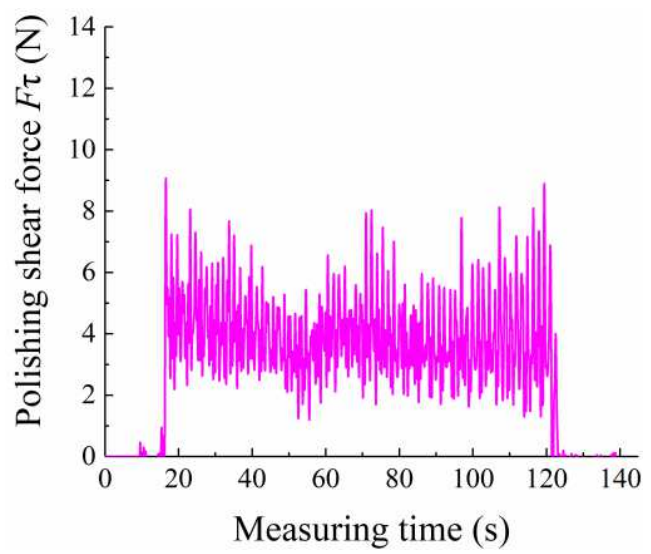

(b) $\varnothing 1 \mathrm{~mm}$

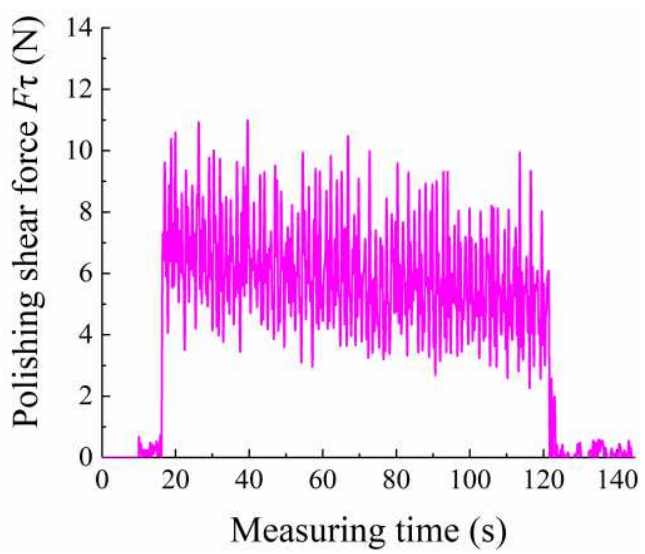

(d) $\varnothing 3 \mathrm{~mm}$

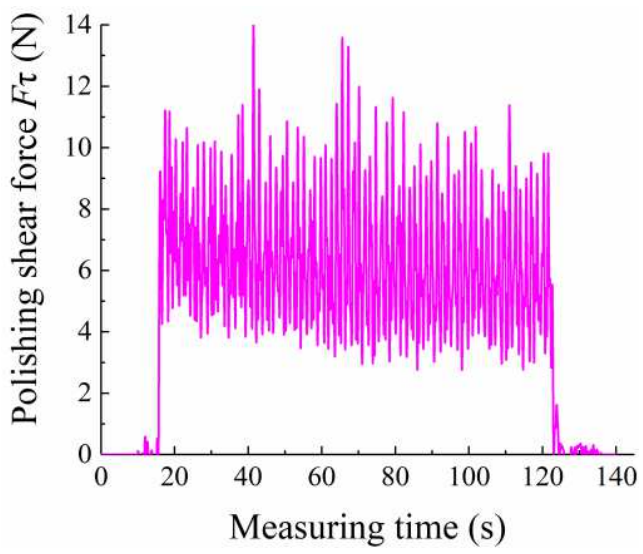

(f) $\varnothing 5 \mathrm{~mm}$

Fig. 7 Influence of different circular hole diameters on polishing shear force

Comparing the measured results of the polishing shear force with the theoretical values, as shown in Fig. 8, it can be seen that the numerical values of the average shear force are in good agreement with the experimental values, when the circular hole diameter is $\varnothing 0 \mathrm{~mm}, \varnothing 1 \mathrm{~mm}, \varnothing 2 \mathrm{~mm}, \varnothing 3 \mathrm{~mm}, \varnothing 4 \mathrm{~mm}$ and $\varnothing 5 \mathrm{~mm}$. The corresponding errors between theoretical calculation values and experimental measurements are $10.2 \%, 6.9 \%, 6.7 \%, 8.6 \%, 8.8 \%$ and $7.6 \%$, respectively. Under the conditions of these parameters, there is a good degree of fit, indicating that the established model can be used to predict the polishing shear force quantitatively. When the circular hole diameter is $4 \mathrm{~mm}$, the error between theoretical calculation and experimental measurement is the largest, which is $8.8 \%$. These errors are mainly caused by the assumption of the idealized distribution of carbonyl iron powder and 
abrasives particles. The relative sliding between the polishing pad and the polishing disk surface slows down, but there is a slow relative sliding, which will lead to a small polishing shear force measurement result and increase the error; secondly, equipment factors such as equipment vibration and dynamometer noise also have a corresponding impact on the error.

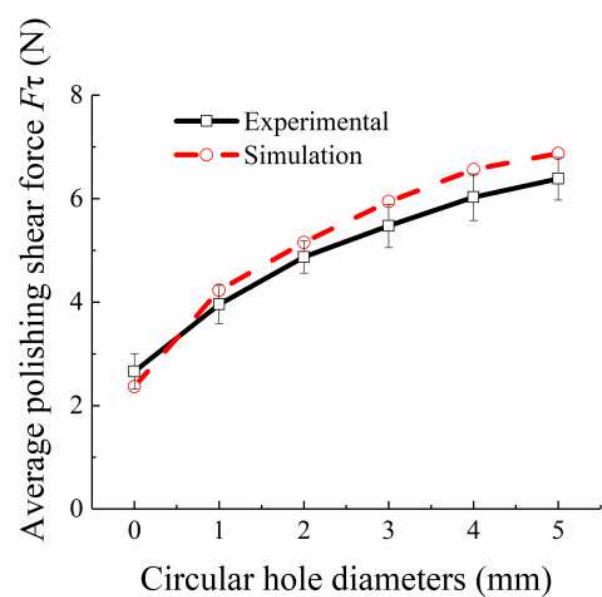

Fig. 8 Comparison between experimental measurement and theoretical calculation of polishing shear force under different circular hole diameters

\subsection{Influence of circular hole diameter on polishing effect}

In order to verify the correctness of the material removal rate model established above, $180 \mathrm{~min}$ polishing experiments of single-crystal sapphire were carried out with different circular hole diameters under the condition of the circular hole of $10 \mathrm{~mm}$ and circular hole depth of $2 \mathrm{~mm}$. The experimental measurement and theoretical calculation results of material removal rate with different circular hole diameters are shown in Fig. 9. It can be seen from Fig. 9 that the theoretical calculation value and the experimental measurement value of material removal rate gradually increase, and the growth slows down in the position of large circular hole diameter. The theoretical value is consistent with the experimental value, the maximum deviation is $10.8 \%$, and the degree of fit is good.
Figure 10 shows the influence of circular hole diameter on the polishing effect of sapphire. When the circular hole diameters are $\varnothing 0 \mathrm{~mm}, \varnothing 1 \mathrm{~mm}, \varnothing$ $2 \mathrm{~mm}, \varnothing 3 \mathrm{~mm}, \varnothing 4 \mathrm{~mm}, \varnothing 5 \mathrm{~mm}$, the corresponding surface roughness is $\mathrm{Ra} 1.36 \mathrm{~nm}, \mathrm{Ra} 0.83 \mathrm{~nm}, \mathrm{Ra}$ $0.76 \mathrm{~nm}, \mathrm{Ra} 1.23 \mathrm{~nm}$, Ra $1.52 \mathrm{~nm}$, Ra $1.68 \mathrm{~nm}$; the material removal rates are $4.37 \mathrm{~nm} / \mathrm{min}, 7.82$ $\mathrm{nm} / \mathrm{min}, 9.53 \mathrm{~nm} / \mathrm{min}, 1.10 \mathrm{~nm} / \mathrm{min}, 12.14 \mathrm{~nm} / \mathrm{min}$, $12.71 \mathrm{~nm} / \mathrm{min}$, respectively. As shown in Fig. 10, with the increase of circular hole diameter, the surface roughness first decreases and then increases, and the material removal rate increases gradually. When the circular hole diameter is $2 \mathrm{~mm}$, the lowest surface roughness reaches $\mathrm{Ra} 0.76 \mathrm{~nm}$, as shown in Fig. 10. When the circular hole diameter is small, the polishing force and material removal rate of the polishing pad on the sapphire surface is low. The unremoved scratches and pits lead to a poor polishing effect. With the circular hole diameter increase, the polishing force and material removal rate increase. The number of pits and scratches on the workpiece surface decreases gradually, showing a minor surface roughness. When the circular hole diameter is too large, the material removal efficiency is too large, there will be apparent removal texture on the workpiece surface, and the surface roughness is more significant. When the circular hole diameter reaches $5 \mathrm{~mm}$, the polishing force of the polishing pad on the workpiece surface is too large, a large number of shallow new scratches occur on the sapphire surface, and the surface roughness increases to $\mathrm{Ra} 1.92 \mathrm{~nm}$. 


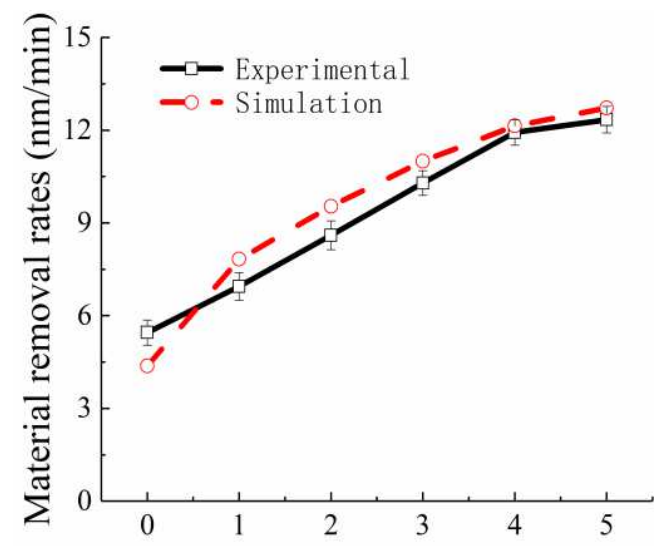

Circular hole diameters ( $\mathrm{mm}$ )

Fig. 9 Comparison between experimental measurement and theoretical calculation of material removal rate under different circular hole diameters

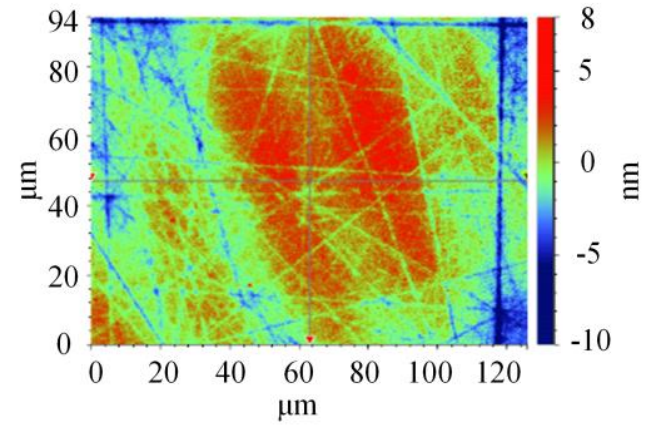

(a) $\varnothing 0 \mathrm{~mm}$

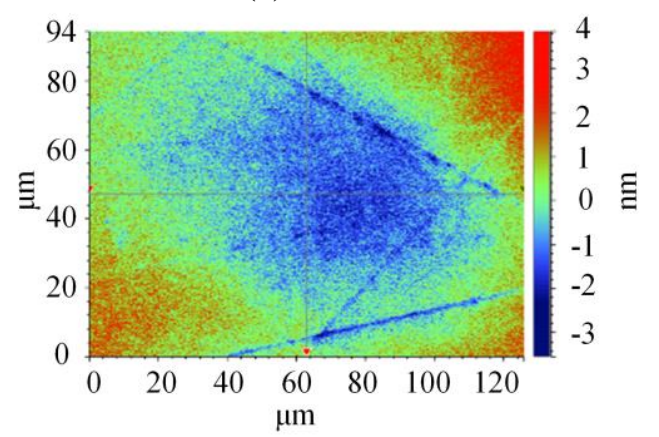

(b) Ø $1 \mathrm{~mm}$

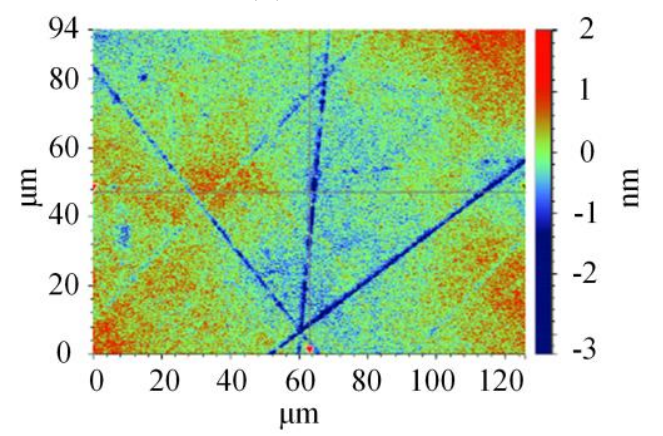

(c) $\emptyset 2 \mathrm{~mm}$

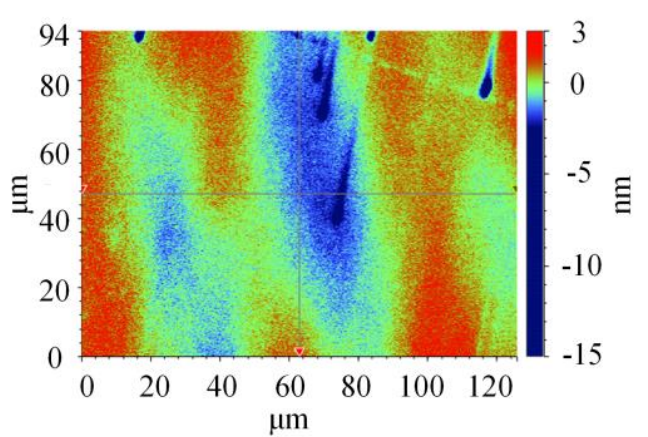

(d) $\varnothing 3 \mathrm{~mm}$

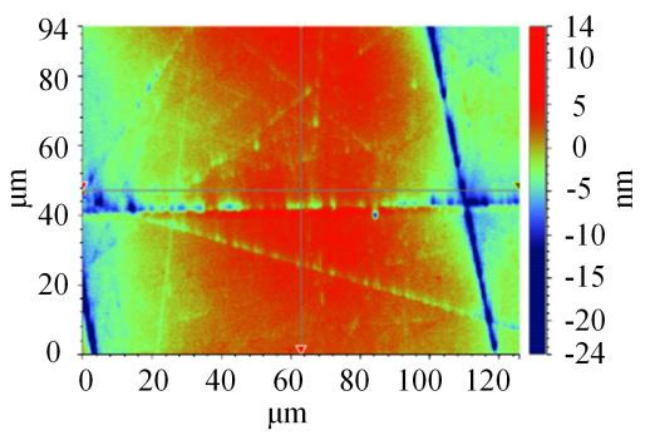

(e) $\varnothing 4 \mathrm{~mm}$

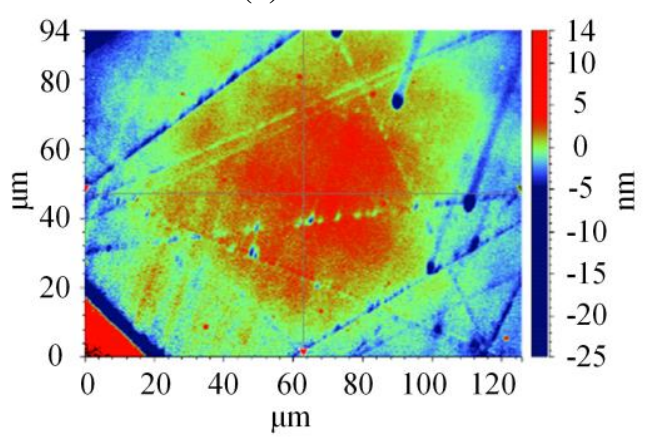

(f) $\varnothing 5 \mathrm{~mm}$

Fig. 10 Influence of circular hole diameter on surface morphology of workpiece

\section{Conclusions}

In this paper, a new cluster MRF method based on array circular holes polishing disk is proposed. In order to better guide the optimization of polishing disk surface structure and processing parameters, the prediction model of polishing shear force and material removal rate of this method is established. The theoretical calculation is carried out through the above two models and verified by rotary dynamometer and polishing tests. The main conclusions are as follows:

(1) In the cluster MRF based on array circular holes polishing disk, based on the contact principle of solid particles, the calculation equation of polishing shear stress of carbonyl iron powder and 
abrasive particles in the polishing pad is deduced theoretically, and the shear force model of solid particles is established.

(2) According to the polishing shear stress model of solid particles, the calculation model of polishing shear force on the workpiece surface is established. Through the actual force measurement of the rotary dynamometer, the effectiveness of the polishing shear force model is verified. When the circular hole diameter is $4 \mathrm{~mm}$, the error between theoretical calculation and experimental measurement is the largest, which is $8.8 \%$.

(3) According to the Preston equation, the material removal model of the cluster MRF method based on array circular holes polishing disk is established. The correctness of the model for predicting material removal rate is verified by polishing experiments with different circular holes, and the maximum deviation is $10.8 \%$. The degree of fit is good.

(4) With the increase of the circular hole diameter, the polishing shear force on the workpiece surface increases gradually. When the circular hole diameter is $5 \mathrm{~mm}$, the polishing shear force increases the most, which is $139.8 \%$ higher than that of the traditional cluster MRF. With the increase of circular holes diameter, the surface roughness decreases at first and then increases, and the material removal rate increases gradually. When the circular hole diameter is $2 \mathrm{~mm}$, the lowest surface roughness reaches $\mathrm{Ra}$ $0.76 \mathrm{~nm}$.

\section{Acknowledgments}

This work was supported by the NSFC-Guangdong Joint Fund Project of China (No. U1801259).

\section{Compliance with ethical standards}

1. The material has not been published in whole or in part elsewhere;

2. The paper is not currently being considered for publication elsewhere;
3. All authors have been personally and actively involved in substantive work leading to the report, and will hold themselves jointly and individually responsible for its content;

4. The authors declare that they have no conflict of interest.

\section{References}

[1] Xu YC, Lu J, Xu XP, Chen CCA, Lin YJ (2018) Study on high efficient sapphire wafer processing by coupling SG-mechanical polishing and GLA-CMP. Int J Mach Tool Manu. 130 131: https://doi.org/10.1016/j.ijmachtools.2018.03.00 2.

[2] Wan SL, Wei CY, Hu C, Situ GH, Shao YC, Shao JD 2021 Novel magic angle-step state and mechanism for restraining the path ripple of magnetorheological finishing. Int $\mathbf{J}$ Mach Tool Manu. https://doi.org/10.1016/j.ijmachtools.2020.10367 3.

[3] Ghosh G, Sidpara A, Bandyopadhyay PP 2021 Experimental and theoretical investigation into surface roughness and residual stress in magnetorheological finishing of OFHC copper. J Mater Process Tech. 288:116899. https://doi.org/10.1016/j.jmatprotec.2020.116899

[4] Meng N, Cao JG, Li JY Fu MH 2019 Magnet arrangements in a magnetic field generator for magnetorheological finishing. Int $\mathrm{J}$ Mech Sci, 161-162:

105018 https://doi.org/10.1016/j.ijmecsci.2019.105018.

[5] Luo H, Guo MJ, Yin SH, Chen FJ, Huang S, Lu A, Guo YF 2018 An atomic-scale and high efficiency finishing method of zirconia ceramics by using magnetorheological finishing. Appl Surf Sci 444:569-577. https://doi.org/10.1016/j.apsusc.2018.03.091. 
[6] Wu JZ, Yin SH, Yang SJ, Guo YF 2020 study on magnetorheological nano-polishing using lowfrequency alternating magnetic field. Adv Mech Eng,

12(1):1-11. https://doi.org/10.1177/1687814019900721.

[7] Guo HR, Wu YB, Lu D Fujimoto M, Nomura M 2014 Effects of pressure and shear stress on material removal rate in ultra-fine polishing of optical glass with magnetic compound fluid slurry. J Mater Process Tech. 214(11): 27592769.

https://doi.org/10.1016/j.jmatprotec.2014.06.014

[8] Xiu SC, Wang RS, Sun BW, Ma L, Song WL 2018 Preparation and experiment of magnetorheological polishing fluid in reciprocating magnetorheological polishing process. J Intel Mat Syst Str. 29 (1) 125-136. https://doi.org/10.1177/1045389X17698247.

[9] Xu JH, Li JY, Liu YM 2021 Investigation on the normal force in cluster magnetorheologicalporous foam finishing process. Tribol Int. 157:106911.

https://doi.org/10.1016/j.triboint.2021.106911.

[10] Zhai Q, Zhai WJ, Gao B 2021 Modeling of forces and material removal rate in ultrasound assisted magnetorheological polishing (UAMP) of sapphire. Colloid Surface A. 628(2):127272. https://doi.org/10.1016/j.colsurfa.2021.127272.

[11] Ghai V, Ranjan P, Batish A, Singh H 2018 Atomic-level finishing of aluminum alloy by chemo-mechanical magnetorheological finishing (CMMRF) for optical applications $\mathrm{J}$ Mater Process Tech. 32:635-643. https://doi.org/10.1016/j.jmapro.2018.03.032.

[12] Yu P, Pan JS, Yan QS, Li WH 2017 An experimental analysis of strontium titanate ceramic substrates polished by magnetorheological finishing with dynamic magnetic fields formed by rotating magnetic poles. Smart Mater Struct 26:055017. https://doi.org/10.1088/1361-665X/aa67cf.

[13] Pan JS, Yan QS 2015 Material removal mechanism of cluster magnetorheological effect in plane polishing. Int $\mathrm{J}$ Adv Manuf Tech. 81: 2017-26. https://doi.org/10.1007/s00170-0157332-7.

[14] Liang HZ, Yan QS, Lu JB, Luo B, Xiao XL 2019 Material removal mechanisms in chemicalmagnetorheological compound finishing. Int $\mathrm{J}$ Adv Manuf Tech. 103: 1337-1348. https://doi.org/10.1007/s00170-019-03594-5.

[15] Luo B, Yan QS, Huang ZL, Pan JS, Fu YZ 2020 Machining method for controlling the behaviours of Bingham fluids in cluster magnetorheological polishing pads. Smart Mater Struct. 30(2): 025002. https://doi.org/10.1088/1361$665 X / a b c d 6 d$.

[16] López-López M, Kuzhir P, Hernández JC 2012 Yield stress in magnetorheological suspensions near the limit of maximum-packing fraction. J Rheol 56(5):

1209. https://doi.org/10.1122/1.4731659.

[17] Hegger C, Maas J 2016 Investigation of the squeeze strengthening effect in shear mode. J Intel Mat Syst Str. 27(14): 1895-1907. https://doi.org/10.1177/1045389X15606998.

[18] Jayswal SC, Jain VK, Dixit PM 2005 Modeling and simulation of magnetic abrasive finishing process. Int J Adv Manuf Tech. 2005, 26(5-6): 477-490. https://doi.org/10.1007/s00170-0042180-x.

[19] Zhang J, Wang H, Kumar AS, Jin MS 2020 Experimental and theoretical Study of internal finishing by a novel magnetically driven polishing tool. Int $\mathrm{J}$ Mach Tool Manu. 153: 103552. https://doi.org/10.1016/j.ijmachtools.2020.10355 2. 
[20] Shizhu W (2012) Pinciples Of Tribology.

Singapore: Tsinghua University Press.

[21] Berman A, Israelachvili JN 2001 Microtribology and micro rheology of molecularly thin liquid film. In: Bhushan B Modern Tribology Handbook. Florida: CRC Press LLC, 2001.

[22] Miao CL, Shafrir SN, Lambropoulos JC, Mici J, Jacobs SD 2009 Shear stress in magnetorheological finishing for glasses. Appl Optics 2585-2594. https://doi.org/10.1364/AO.48.002585 